\title{
Design and Research of the Crane Anti-collision Alarming Device against HV Transmission Wires
}

\author{
Tiao Liu ${ }^{a}$, Xianglian $\mathrm{Xu}^{\mathrm{b}}$, Ting $\mathrm{Liu}^{\mathrm{c}}$, Gang Huang ${ }^{\mathrm{d}}$ and Yuchen Wang ${ }^{\mathrm{e}}$ \\ School of Automation, Wuhan University of Technology, Wuhan City, Hubei Province, China \\ aliutiao@whut.edu.cn, ${ }^{b} x u x i a n g l i a n @ 163 . c o m,{ }^{c}$ natay2010@163.com
}

Keywords: high-voltage transmission wire; anti-collision; sound and light alarm; STM32; NRF905.

\begin{abstract}
The design and research of the crane anti-collision alarming device against HV transmission wires are introduced in this paper. The alarming device can receive wirelessly all the signals sent by the various electric-field sensors of the crane, and then the device can give out different indications about safety, precaution, high-voltage danger and distance warning accordingly so as to protect the high-voltage transmission wire from careless damage of the working cranes.
\end{abstract}

\section{Introduction}

In recent years, short circuit tripping accidents caused by high-altitude construction machinery near high-voltage wires often happened in many provinces. According to incomplete statistics, more than one hundred accident caused by cranes touching the high-voltage wires happened a year. Some caused blackouts in large area, and the national economy suffered severe losses [1-2]. That has brought great influence to people's life and social activities. Therefore, it is important to research how to effectively prevent the accident caused by the crane to touch the high voltage wire.

\section{Research statuses}

In the research of the crane anti-collision alarming device against $\mathrm{HV}$ transmission wires, there are few studies about the application of anti-collision device in the crane construction work near high-voltage power. Most of the anti-collision measures are just to develop safety scheme in detail before construction, for example, to limit tower crane to rotate in only one direction, to set the bamboo-protective barriers [3-4]. Obviously, this approach is time-consuming and laborious. It is not suitable for most of the construction work of crane. There are two types of the crane anti-collision alarming device against $\mathrm{HV}$ transmission wires in China. One of them is infrared detection, obtaining the position and state of the crane [5]. Another is electric induction, determining the risk by inducing the electric field around the high-voltage wire.

\section{System design}

The crane anti-collision alarming device against HV transmission wires described in this paper is designed based on the principle that metal produce surface induced charge in an electric field. The charges of metal surface can converted into the voltage signal. After the election of $50 \mathrm{~Hz}$ frequency filter circuit, the signal amplifying circuit, the signal is sampled by the STM32 microcontroller. The results of the measurements are converted into distance information. The alarm information is transmitted to the alarming host by the wireless communication module NRF905. The host can remind the operator of the danger of high-voltage lines through the sound and light. The whole system is divided into signal processing module, signal acquisition module, wireless transmission module and alarm module. The system frame diagram is shown in figure 1. 


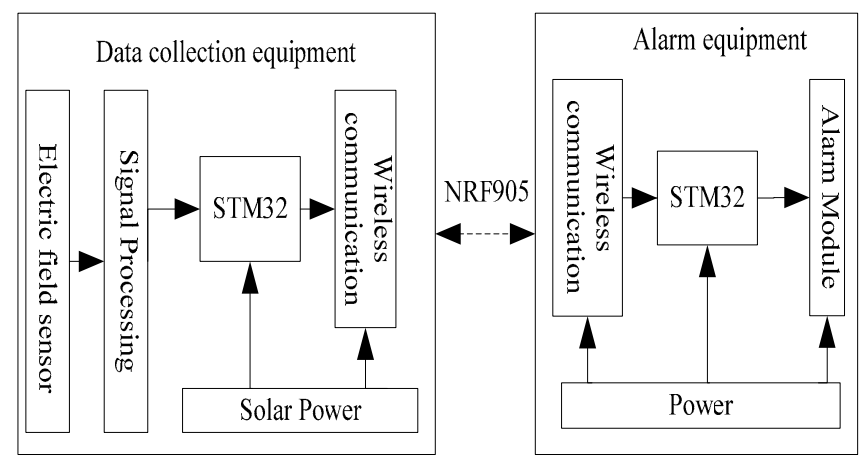

Fig. 1. The system frame diagram

The signal processing module. The signal processing module is the key part of the whole equipment. It is the electric field sensor before the signal processing circuit. The design of the spherical electric field sensor is complex and difficult to process. So a parallel plate is used to be an electric field sensor. A parallel plate capacitor is chosen as a power frequency electric field sensor in this paper. The PCB board is used as the electric field probe in this paper. The equivalent model of the parallel plate sensor is shown in figure 2.

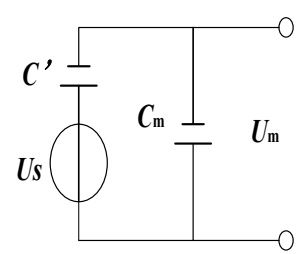

Fig. 2. The equivalent model of the parallel plate sensor

In the actual measurement, the capacitance $C_{m}$ need parallel between the two polar plates except the inherent capacitance $C^{\prime}$ between parallel plates. The electric field strength can be measured by measuring the voltage across the capacitor after accessing the measurement capacitance $\mathrm{C}_{\mathrm{m}}$.

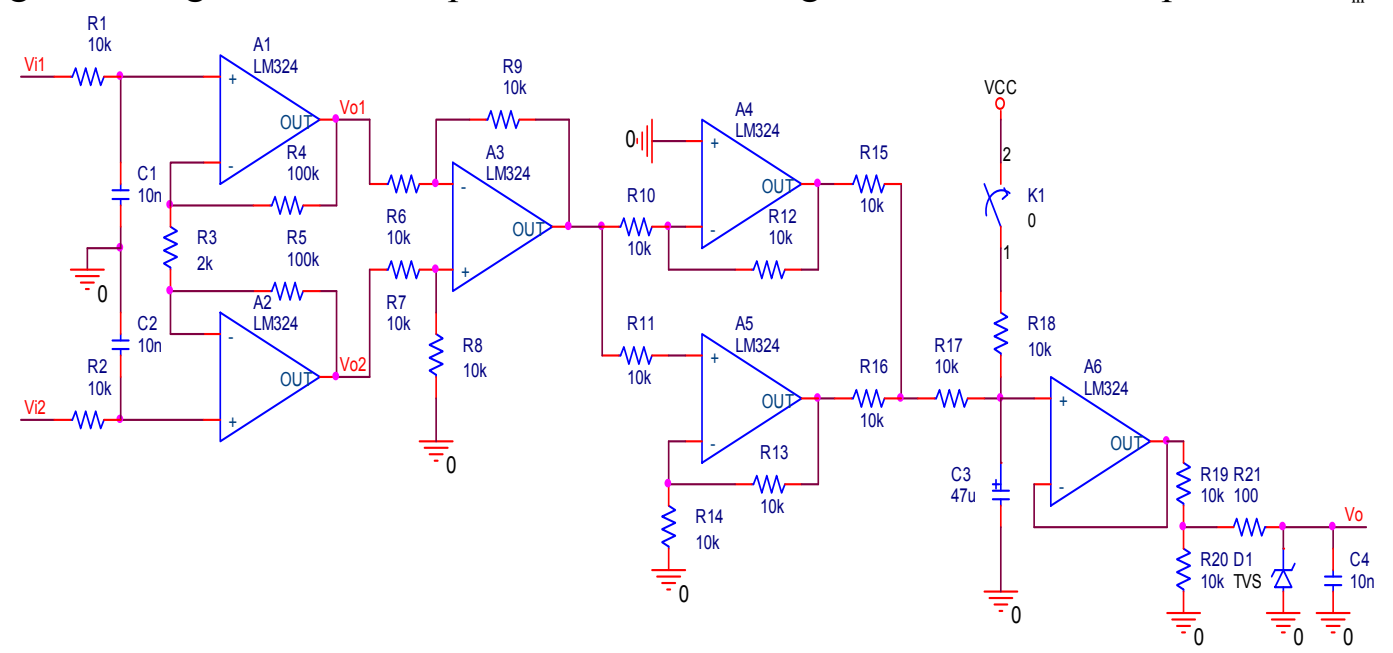

Fig. 3. The signal processing circuit

In the signal processing circuit shown in figure 3, the low pass filters are made of R1、R2、 C1、C2, which can filter the collected voltage signal. As the first stage amplifier connected a phase scale operation circuit, the amplifier of A1 and A2 can amplify the signal. The amplification is:

$\frac{\mathrm{U}_{\mathrm{o} 1}-\mathrm{U}_{\mathrm{o} 2}}{\mathrm{U}_{\mathrm{i} 1}-\mathrm{U}_{\mathrm{i} 2}}=\frac{\mathrm{R}_{3}+\mathrm{R}_{4}+\mathrm{R}_{5}}{\mathrm{R}_{3}}=101$

As the second ratio of differential arithmetic circuit, the amplifier of A3 can increase the CMRR. As the third inverse proportion of operation circuit, the amplifier of A4 andA5 can convert the AC signal into a DC signal. As the signal level fourth follower, the amplifier of A6 can isolate the interference signal. 
The signal acquisition module. This system is based on ARM Cortex-M3 series CPU chip STM32F103C8 core, which consists of the acquisition module. STM32F103C8 chip is a fully integrated low-power mixed-signal system-type MCU. All devices offer two 12-bit ADCs. Signal acquisition module receives a DC voltage signal and processing digital signal. It uses 12 bits of AD, accuracy of $0.0008 \mathrm{~V}$.

Wireless communication module. Wireless communication module uses the Norwegian Nordic single chip RF transmitter chip NRF905. The NRF905 meets the national standard wireless management committee, don't need to apply for the frequency point and works on 433/868/915 MHZ ISM channel (industrial, scientific and medical). The NRF905 can automatically handles preamble and CRC. Configuration is easily programmable by use of the SPI interface. Current consumption is very low, in transmit only $9 \mathrm{~mA}$ at an output power of $-10 \mathrm{dBm}$, and in receive mode $12.5 \mathrm{~mA}$.

Sound and light alarm module. Sound and light alarm module mainly refers to the crane anti-collision alarming device through wireless modules NRF905 which receives the processing signal of the power supply voltage and electric field strength value. The voltage of the crane anti-collision alarming device is $12 \mathrm{VDC}$. When the power supply voltage is lower than $7.5 \mathrm{~V}$, the device can't work and the power light will turn red and flashing, meanwhile the buzzer will give an alarm in a certain frequency. The device can work normally only when the voltage of the device is above $7.5 \mathrm{~V}$. When the electric field strength value is lower than $2 \mathrm{kV} / \mathrm{m}$, the device will not alarm. When the electric field strength value is higher than $2 \mathrm{kV} / \mathrm{m}$ and lower than $4 \mathrm{kV} / \mathrm{m}$, the alarm light of the device will turns into green. When the electric field strength value is higher than $4 \mathrm{kV} / \mathrm{m}$ and lower than $5 \mathrm{kV} / \mathrm{m}$, the alarm light of the device will turns into yellow. When the electric field strength value is higher than $5 \mathrm{kV} / \mathrm{m}$, the alarm light of the device will turns into red. When each light is alarming, the buzzer gives an alarm in different frequency.

Table 1 Field measurement calibration table

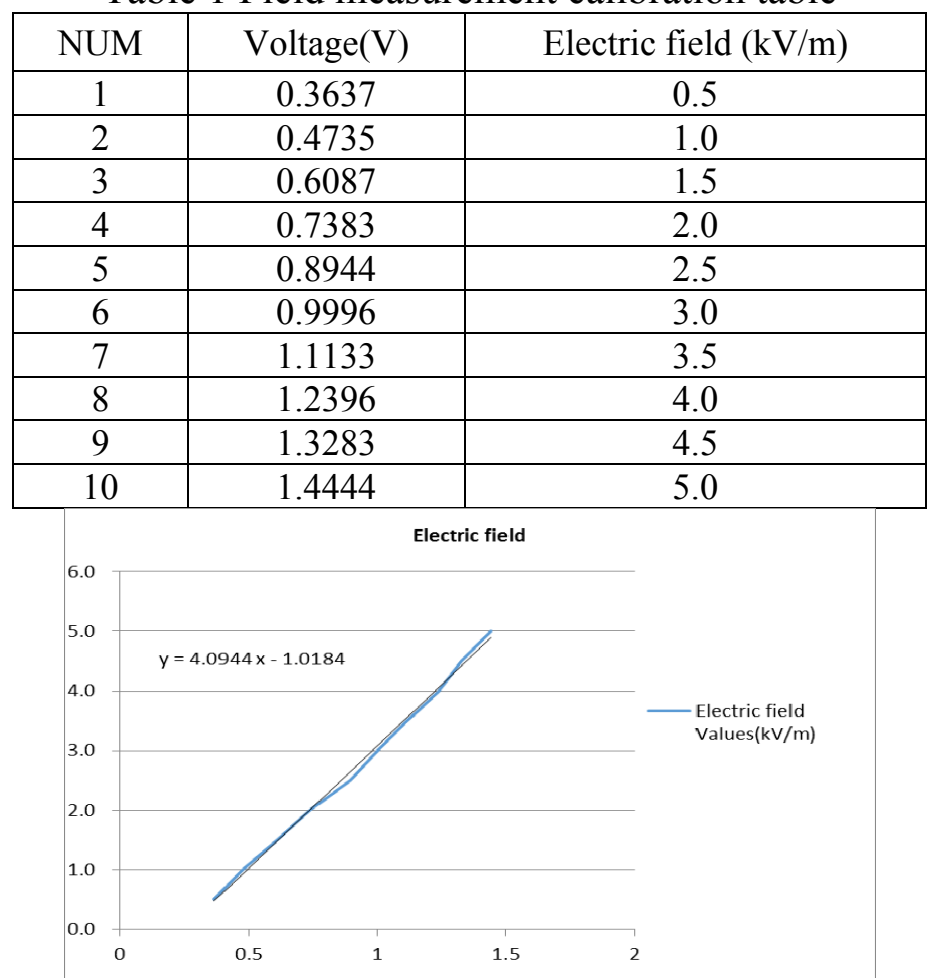

Fig.4. Field measurement calibration table

Table 1 and figure 4 shows the system calibration formula is: $\mathrm{y}=4.0944 \mathrm{x}-1.0184$, where $\mathrm{x}$ is indicated to monitor the parallel plate voltage value, $y$ that the monitoring of the electric field strength. In order to ensure the stability and accuracy of test instrumentation when it works in high voltage and strong electric field environment, we put the anti-collision alarming system of crane and the Narda's EFA300 which is a standard low frequency electromagnetic measuring instrument in a comparison testing. 
Measurement data are shown in table 2 and figure 5. Test data for the two instruments indicates the maximum relative error is $5.56 \%$.And the measurement result is under highly matching. We can conclude that the device presented in this paper meets the requirement of accuracy.

Table 2 The relative error of the measurement table

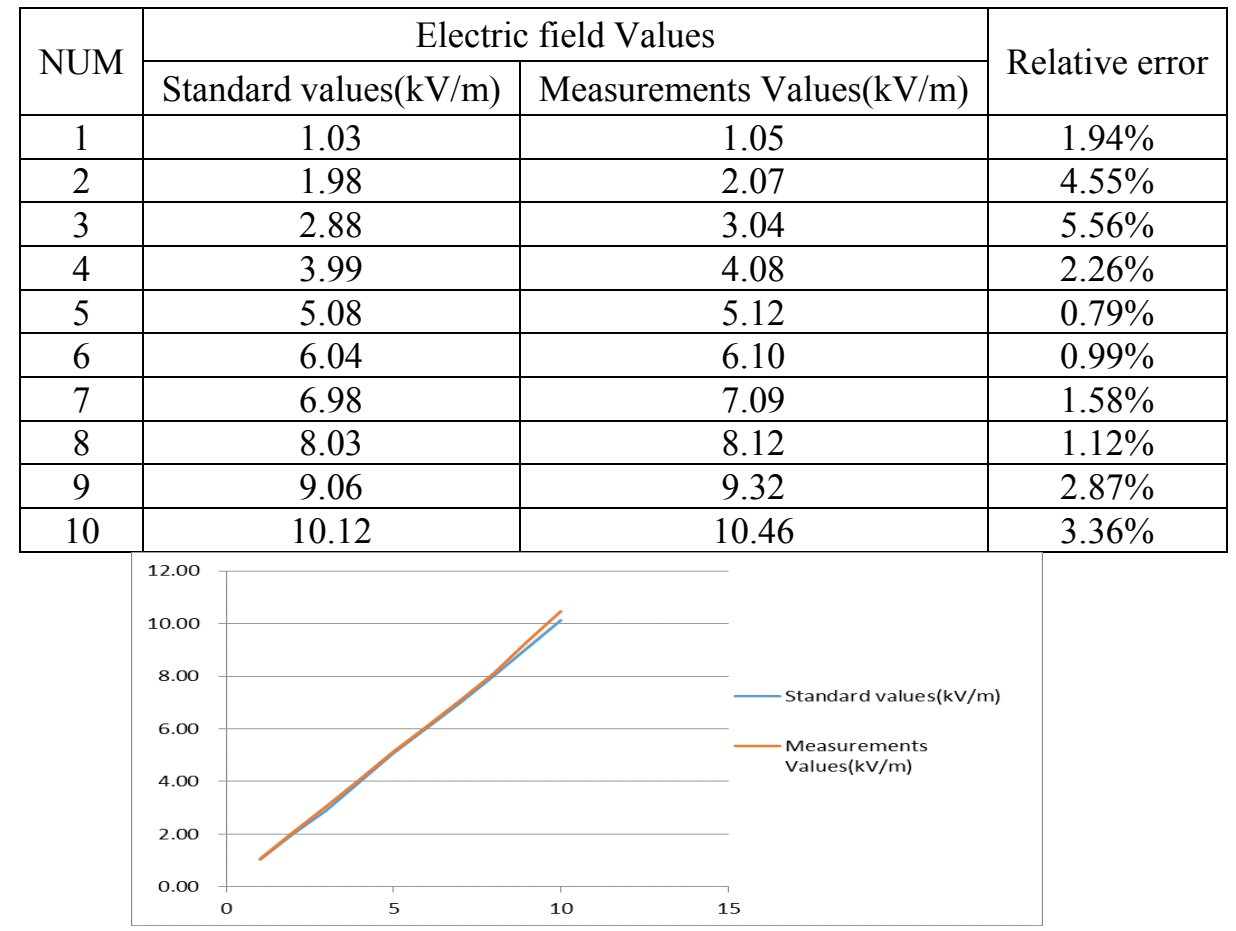

Fig 5 the relative error of the measurement diagram

\section{Summary}

The crane anti-collision alarming device designed in this paper has the characteristics of high precision, small size, practical value, and so on. This device can protect the high voltage transmission wire from careless damage of the working crane. It will have a good prospect.

\section{Acknowledgements}

I'd like to express my gratitude to the corporation of Wuhan NARI. And I want to thank them for giving me the opportunity to design the device.

\section{References}

[1] Jiang Shao Zhen. The high-voltage wire crane anti-collision system detection technology [J]. Electronic test, 2013, 19:155-156.

[2] Guo Hailin, Wang Zhining, Liu Xiao, Zhang Lixin. High voltage AC transmission line power frequency electric field intensity calculation formula of the improvement of [J]. Science technology and engineering, 2014, 30:156-159.

[3] Zhang Xiaolei, Liu Zhenhai, Gao Ming, Qin pine. Alarm device to prevent development of crane touch the high voltage transmission line, [J]. East China Electric Power 2011, 07:1201-1202.

[4] Ding Yueming. High voltage construction safety distance alarm new technology research and prototype development of [D]. Huazhong University of Science and Technology, 2011

[5] Hu Zewen, Yao Degui, Wang Jingang, Ho, Wen Jun, Li Luo. [J]. research on high voltage power frequency electric field warning instrument, Measurement and instrumentation, 2009,09:45-48. 